\title{
Efeito dos bagaços de malte e cana-de-açúcar na retenção de nutrientes em substratos de telhado verde extensivo
}

\author{
Effect of malt and sugar cane bagasse in nutrient retention in extensive green roof substrates
}

Efecto de los bagazos de malta y caña de azúcar en la retención de nutrientes en sustratos de techo verde extensivo

Recebido: 12/02/2021 | Revisado: 20/02/2021 | Aceito: 23/02/2021 | Publicado: 03/03/2021

\author{
Marcella Regyna do Nascimento Scarassatti \\ ORCID: https://orcid.org/0000-0002-5549-3717 \\ Universidade Federal de Mato Grosso, Brasil \\ E-mail: marcella010293@gmail.com \\ Aline Benatti \\ ORCID: https://orcid.org/0000-0003-1778-0376 \\ Universidade Federal do Paraná, Brasil \\ E-mail: benatti.line@gmail.com \\ Eduardo Beraldo de Morais \\ ORCID: https://orcid.org/0000-0002-8505-4133 \\ Universidade Federal de Mato Grosso, Brasil \\ E-mail: beraldo_morais@yahoo.com.br
}

\begin{abstract}
Resumo
Este estudo objetivou avaliar a qualidade da água escoada por unidades experimentais de telhados verdes contendo substratos acrescidos de materiais adsorventes de baixo custo, como o bagaço de malte (M) e o bagaço de cana-deaçúcar (C). Cada unidade experimental foi composta por manta do tipo geotêxtil, um substrato e a vegetação (Arachis repens). Os substratos $\mathrm{M}$ e $\mathrm{C}$ foram comparados com um substrato controle (CON) de grande utilização comercial. O experimento foi desenvolvido em duas etapas, irrigação manual e chuva real. A qualidade da água escoada pelas unidades experimentais foi analisada por meio dos parâmetros de cor verdadeira, turbidez, sólidos totais, $\mathrm{pH}$, condutividade elétrica, nitrato $\left(\mathrm{NO}_{3}^{-}\right)$, fosfato $\left(\mathrm{PO}_{4}^{3-}\right)$ e sulfato $\left(\mathrm{SO}_{4}^{2-}\right)$. As diferenças nos substratos exerceram efeitos significativos nas concentrações de $\mathrm{NO}_{3}^{-}$e $\mathrm{SO}_{4}^{2-}$, sendo o substrato $\mathrm{C}$ com melhor desempenho na retenção destes ânions. A distribuição e o impacto da água nas unidades experimentais apresentaram diferenças quanto ao carreamento de nutrientes, com menor lixiviação na fase de irrigação manual. Os resultados sugerem que a aplicação do bagaço de cana-de-açúcar em telhados verdes para a melhoria da qualidade da água escoada é promissora.
\end{abstract}

Palavras-chave: Coberturas verdes; Qualidade da água; Materiais de baixo custo.

\begin{abstract}
This study aimed to evaluate the quality of runoff water of experimental units of green roofs containing substrates added of low-cost adsorbent materials, such as malt bagasse (M) and sugar cane bagasse (C). Each experimental unit was built with a geotextile membrane, a substrate, and vegetation (Arachis repens). The substrates $\mathrm{M}$ and $\mathrm{C}$ were compared with a control substrate (CON) widely used commercially. The experiment was developed in two steps: manual irrigation and rainfall. The quality of the runoff water from the experimental units was analyzed using the parameters real color, turbidity, total solids, $\mathrm{pH}$, electric conductivity, nitrate $\left(\mathrm{NO}_{3}^{-}\right)$, phosphate $\left(\mathrm{PO}_{4}^{3-}\right)$ and sulphate $\left(\mathrm{SO}_{4}^{2-}\right)$. The substrates had relevant effects in the concentrations of $\mathrm{NO}_{3}^{-}$, and $\mathrm{SO}_{4}^{2-}$, being that the substrate $\mathrm{C}$ had better performance retention. Water distribution and impact in the experimental units presented differences regarding nutrients transport, with less lixiviate in the manual irrigation step. The results suggest that using sugar cane bagasse in green roofs to improve runoff quality is promising.
\end{abstract}

Keywords: Green roof; Water quality; Low-cost materials.

\section{Resumen}

Este estudio tuvo como objetivo evaluar la calidad del agua drenada por unidades experimentales de techos verdes que contienen sustratos más materiales adsorbentes de bajo costo, como el bagazo de malta (M) y el bagazo de caña de azúcar (C). Cada unidad experimental fue compuesta por una manta geotextil, un substrato y la vegetación (Arachis repens). Los substratos $\mathrm{M}$ y $\mathrm{C}$ fueron comparados con un sustrato control (CON) largamente utilizado en el comercio. El experimento fue desarrollado en dos etapas: irrigación a mano y lluvia real. La calidad del agua drenada en las 
unidades experimentales fue analizada con los parámetros color verdadero, turbidez, solidos totales, $\mathrm{pH}$, conductividad eléctrica, nitrato $\left(\mathrm{NO}_{3}^{-}\right)$, fosfato $\left(\mathrm{PO}_{4}^{3-}\right)$ y sulfato $\left(\mathrm{SO}_{4}^{2-}\right)$. Las diferencias en los sustratos tuvieron efectos significativos en las concentraciones de $\mathrm{NO}_{3}^{-}, \mathrm{y} \mathrm{SO} \mathrm{SO}_{4}^{2-}$, y el sustrato $\mathrm{C}$ tuvo mejor desempeño em la retención de estos aniones. La distribución y el impacto del agua en las unidades experimentales presentaron diferencias en el transporte de nutrientes, con menos lixiviación en la etapa de irrigación a mano. Los resultados sugieren que la aplicación de bagazos de caña de azúcar en techos verdes para mejorar la calidad del agua es prometedora.

Palabras clave: Techos verdes; Calidad del agua; Materiales de bajo costo.

\section{Introdução}

O crescimento exacerbado dos centros urbanos tem tornado as medidas indutoras da proteção do ambiente, aliadas ao desenvolvimento sustentável, cada vez mais necessárias. O interesse público e de pesquisa em telhados verdes aumentaram nos últimos anos, muito provavelmente como resultado da variedade de benefícios ambientais que são frequentemente atribuídos a eles.

Os telhados verdes são ferramentas que podem ser incluídas em estratégias de mitigação de problemas ambientais em áreas urbanizadas, reduzindo o escoamento de águas pluviais (Vijayaraghavan \& Raja, 2014), conservando energia, mitigando ilhas de calor, sequestrando carbono (Wahba et al., 2018; Wahba et al., 2019), fornecendo habitat para a vida selvagem e dando espaço para a produção de alimentos urbanos (Berndtsson, 2010; Rowe, 2011).

Os inúmeros aspectos positivos diretos e indiretos do uso de coberturas verdes demostram a importância econômica e ambiental de sua implantação em ambientes urbanos (Niemelã, 2014). Entretanto, o conhecimento insuficiente sobre os aspectos técnicos e construtivos se torna uma barreira para o seu desenvolvimento e aplicação no Brasil (Noya et. al. 2017). A maior parte das técnicas e materiais utilizados para telhados verdes foi desenvolvida em outros países, onde as condições climáticas são diferentes daquelas encontradas no Brasil.

Os substratos usados nos telhados verdes são a base de um desenvolvimento bem sucedido da vegetação. Os componentes do substrato podem, teoricamente, atuar como adsorventes de poluentes e filtros, tanto quanto, podem contribuir potencialmente para a degradação da qualidade das águas receptoras com poluentes liberados do solo, plantas e fertilizantes (Vijayaraghavan et al., 2012; Vijayaraghavan \& Raja, 2014).

Alguns estudos observaram que os telhados verdes podem ser produtores de poluentes. Alsup et al., 2011 e Speak et al., 2014 apontaram a presença de metais pesados e outros contaminantes no escoamento dos sistemas de telhados verdes, como por exemplo, $\mathrm{Cd}, \mathrm{Cr}, \mathrm{Cu}, \mathrm{Fe}, \mathrm{K}, \mathrm{Mn}, \mathrm{Pb}, \mathrm{Zn}, \mathrm{NO}_{3}^{-}, \mathrm{NH}_{4}^{-}$, N-total, $\mathrm{PO}_{4}^{-}$e P-total. Berndtsson et al. (2006) relataram que, embora em concentrações mais baixas do que as normalmente encontradas no escoamento urbano, alguns metais aparecem em escoamento de telhados verdes em concentrações que corresponderiam a águas naturais moderadamente poluídas.

Por outro lado, alguns estudos observaram que os telhados verdes funcionam como sumidouro de poluentes e podem melhorar qualidade da água pluvial (Berndtsson et al., 2006; Vijayaraghavan \& Joshi 2014; Vijayaraghavan \& Raja, 2014).

Investigar a qualidade dos substratos para utilização em telhados verdes é um fator relevante, pois o desenvolvimento de substratos que forneçam condições mais favoráveis não somente para o crescimento da vegetação, mas também que carreie a menor quantidade de nutrientes possíveis é primordial para a sustentabilidade desse sistema (Young et al., 2014). Como observa Noya et al. (2017), esses substratos podem ser subprodutos de indústrias, a fim de mitigar a produção de resíduos que, se não reutilizados, tornam-se potencial poluente ambiental.

Os resíduos agroindustriais são produzidos em grandes quantidades, a partir do beneficiamento e industrialização de alimentos. Por exemplo, na indústria cervejeira, o bagaço de malte, tem sido objeto de estudo de diversas pesquisas voltadas para as suas capacidades adsortivas, apresentando potencial na remoção de corantes industriais (Reis et al. 2018). Da mesma 
forma, o bagaço de cana-de-açúcar é um subproduto com potencial adsortivo analisado em algumas pesquisas, como na adsorção de metais como $\mathrm{Cu}, \mathrm{Cr}$ e $\mathrm{Pb}$ (Ferreira et al., 2015) e na adsorção de azul de metileno (Silva \& Oliveira, 2012).

Nesse contexto, o objetivo deste trabalho foi investigar se a adição de materiais adsorventes de baixo custo em substratos de telhados verdes extensivos, sendo o bagaço de malte e o bagaço de cana-de-açúcar, alteram a qualidade da água escoada pelos telhados verdes. Para isso, unidades experimentais simulando telhados verdes extensivos foram confeccionadas com uma espécie vegetal (Arachis repens) e três tipos diferentes de substratos. A qualidade da água escoada foi avaliada em duas fases, sendo a primeira durante a irrigação manual das unidades experimentais e a segunda em eventos chuvosos reais.

\section{Metodologia}

\subsection{Caracterização geral da área de estudo}

A pesquisa é caracterizada como experimental de natureza quantitativa (Pereira et al. 2018). O trabalho foi desenvolvido na Universidade Federal de Mato Grosso (UFMT), na latitude $15^{\circ} 36^{\prime} 26.0^{\prime \prime} \mathrm{S}$ e longitude 5603'40.6"W, à aproximadamente 30 metros de distância da Estação Climatológica Mestre Bombled, no município de Cuiabá, MT, mesorregião Centro-Sul-Mato-Grossense. O clima, segundo a classificação climática de Köppen, é do tipo Aw - Clima de Savana, caracterizado como Tropical Semi-úmido.

A Figura 1 apresenta a precipitação média mensal no período de 1989 a 2018, com dados provenientes da Estação Climatológica Mestre Bombled.

Figura 1: Precipitação média mensal registrada no período de 1989 a 2018. Dados obtidos da Estação Climatológica Mestre Bombled - UFMT.

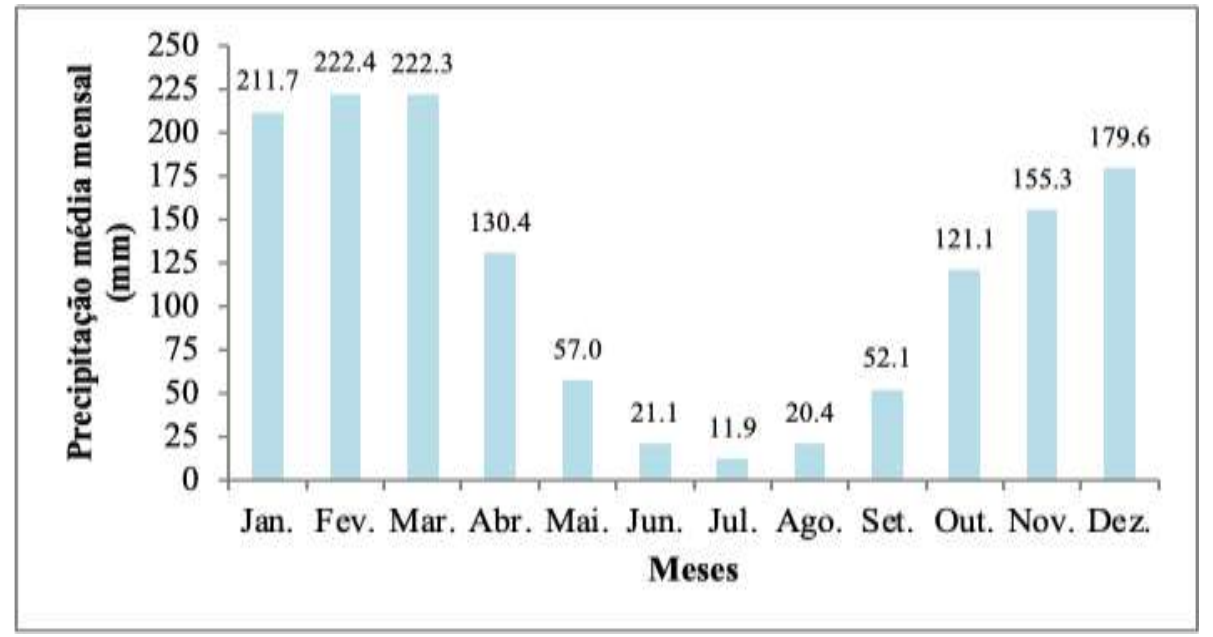

Fonte: Dados obtidos da Estação Climatológica Mestre Bombled - UFMT.

Observa-se na Figura 1 que a região possui sazonalidade marcada por dois períodos bem distintos: a estiagem (abril a setembro) e as chuvas (outubro a março).

A temperatura média anual é de $26^{\circ} \mathrm{C}$, ocorrendo máximas médias diárias em torno de $36{ }^{\circ} \mathrm{C}$, em setembro, e as mínimas de $15^{\circ} \mathrm{C}$, em junho (Mato Grosso, 2016). No período chuvoso, principalmente nos primeiros meses, a temperatura é mais elevada. A umidade do ar mantém-se alta, em torno de 81,3\%. Assim, durante a época das chuvas, o clima é tropical continental úmido. A umidade do ar em certos dias desce a uma porcentagem mínima que varia de 18\% a 40\% (Cuiabá, 2012). 


\subsection{Unidades experimentais}

A unidade experimental apresentava formato quadrado com lado igual a $15 \mathrm{~cm}$, constituídas com fundo falso removível de 2 centímetros de altura e orifícios que simulavam a drenagem de um telhado verde, conforme esquematizado na Figura 2.

Figura 2: Representação da unidade experimental.

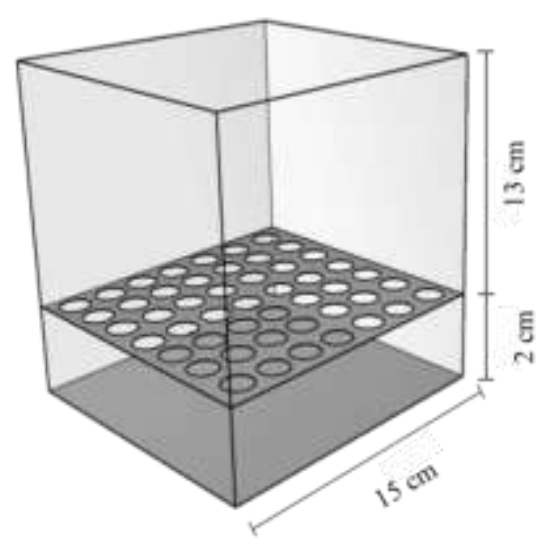

Fonte: Autores.

Essa estrutura removível foi construída para receber a água escoada. O material utilizado para a fabricação dos recipientes foi o acrílico com espessura de $2 \mathrm{~mm}$. Para evitar vazamentos, as estruturas foram reforçadas com cola específica para o material utilizado.

Cada unidade experimental foi composta por manta do tipo geotêxtil modelo Bidim disposta sobre o fundo perfurado da unidade experimental, a qual recebeu o substrato e a vegetação. A manta geotêxtil possuía $10 \mathrm{~mm}$ de espessura e era composta por filamentos de polipropileno e poliamida (nylon), tendo índice de vazios em torno de $95 \%$, de acordo com o fabricante.

O experimento foi desenvolvido em estufa de vegetação, na fase correspondente a irrigação manual (fase 1) e após 90 dias foi avaliado na fase de chuva real (fase 2) detalhada no item 2.5.

\subsection{Seleção e caracterização do substrato}

Foram avaliadas três composições de substratos de telhados verdes, sendo um substrato comercial, considerado o controle do experimento, e esse mesmo substrato adicionado de bagaço de malte e bagaço de cana-de-açúcar. Seguindo a classificação de um telhado verde extensivo, a profundidade do substrato foi definida em 10 centímetros, para todas as unidades experimentais. Os bagaços de malte e de cana-de-açúcar foram misturados separadamente ao substrato comercial, na proporção em volume 1:4, respectivamente. O substrato comercial era composto por esterco de aves com calcário, cascas processadas e decompostas e vermiculita expandida, sendo amplamente utilizado como substrato para jardinagem.

Antes da adição dos bagaços de malte e de cana-de-açúcar ao substrato comercial, os mesmos passaram por um processo de preparação inicial, com a finalidade de eliminar o excesso de açúcares. Primeiramente, os compostos foram abundantemente lavados com água corrente e posteriormente secos à uma temperatura de $70^{\circ} \mathrm{C}$. Em seguida, os compostos foram triturados, selecionando-se partículas de $0,25-2 \mathrm{~mm}$ por meio de peneiras granulométricas com malhas desejadas. Para o substrato comercial, controle, também foram fixadas partículas de granulometria de $0,25-2 \mathrm{~mm}$. 
As propriedades físicas e químicas de cada composição dos substratos, $\mathrm{CON}$ (substrato comercial); C (CON / bagaço de cana-de-açúcar); M (CON / bagaço de malte), foram avaliadas antes do plantio da espécie vegetal. Os parâmetros físicos e químicos dos três tipos de substratos foram mensurados adicionando-se $1 \mathrm{~g}$ da mistura do substrato em $100 \mathrm{~mL}$ de água destilada. O conteúdo foi agitado a $200 \mathrm{rpm}, 30^{\circ} \mathrm{C}$ por $24 \mathrm{~h}$ (Vijayaraghavan \& Raja, 2014). O sobrenadante (solubilizado) foi filtrado a vácuo usando membrana de fibra de vidro e o filtrado foi analisado quanto ao $\mathrm{pH}$, condutividade elétrica (CE), turbidez, cor verdadeira, nitrato, fosfato e sulfato. No caso dos íons analisados, as medições foram efetuadas usando kit de reagentes Hach de acordo com as instruções do fabricante e as leituras foram feitas em espectrofotômetro UV-Vis (modelo DR6000 - Hach) (www.hach.com). Também foram mensuradas a densidade aparente, capacidade de campo e tamanho e massa das partículas do substrato, conforme metodologia descrita no Manual de Métodos de Análise de solos (EMBRAPA, 2017). Analisou-se também a capacidade de troca catiônica (CTC) utilizando os métodos analíticos oficiais para fertilizantes e corretivos (Brasil, 2017).

Os diferentes tipos de substratos foram adicionados nas unidades experimentais de forma manual e sem compactação, até alcançar a profundidade desejada. Para fins de estabilização dos substratos, esses foram mantidos por 30 dias, em temperatura ambiente, no Laboratório de Microbiologia do Departamento de Engenharia Sanitária e Ambiental da Universidade Federal de Mato Grosso. Nesse período, antes do plantio, a cada sete dias os substratos foram irrigados com 0,5 litros de água de torneira sem cloro, para manter a umidade e acelerar os processos biológicos.

\subsection{Seleção e caracterização da vegetação}

Selecionou-se uma vegetação que fosse viável às condições climáticas locais e adaptável à diferentes substratos (Johnston \& Newton, 2004), assim optou-se por utilizar a espécie Arachis repens Handro, conhecida como grama-amendoim.

A grama-amendoim pertencente à família Fabaceae, é uma planta herbácea rasteira, estolonífera, perene e nativa do Brasil, notadamente de regiões de cerrado e costeiras do país (Valls, 1992). Pode alcançar 0,10 a 0,20 m de altura, com ramagem prostrada, resistente ao sol e solos de moderada fertilidade, tolerante à acidez do solo ( $\mathrm{pH}$ em torno de 5) e à alta saturação de alumínio (75\%) (Valls, 1992; Rincón et al., 1992). De acordo com Rincón et al. (1992), tolera também condições de má drenagem e encharcamento temporário. Além destas adaptações, possui ainda benefício por meio de associação simbiótica com bactérias fixadoras de nitrogênio atmosférico do gênero Bradyrhizobium, que infectam as raízes e criam nódulos radiculares, os quais fornecem a maior parte do nitrogênio necessário ao desenvolvimento.

O plantio foi realizado no dia 11/06/2018. Buscou-se selecionar as mudas com maior grau de uniformidade, caracterizando-as quanto à altura do caule e da raiz. Como as estacas foram previamente cultivadas em mistura comercial, o máximo possível dessa mistura foi removido lavando o corte em água antes do plantio para reduzir os efeitos desse meio comercial no substrato do telhado verde.

Em cada unidade experimental foram plantadas 4 mudas. O período de estabelecimento da vegetação foi de 30 dias. A Figura 3 apresenta a imagem das mudas plantadas nas unidades experimentais. 
Figura 3: Unidades experimentais após o plantio da vegetação.

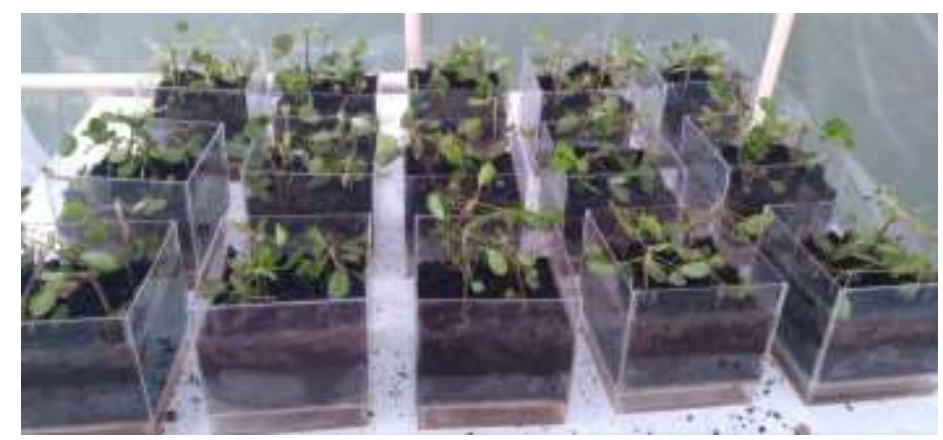

Fonte: Autores.

\subsection{Monitoramento experimental}

O fornecimento de água nas unidades experimentais foi realizado de duas maneiras. No primeiro estágio da pesquisa a entrada de água se deu por irrigação manual, em estufa de vegetação, essa etapa foi denominada de Fase 1. Após 90 dias desde o plantio, os parâmetros da qualidade da água foram analisados por meio de eventos de chuva real denominada Fase 2.

A irrigação foi caracterizada como um tratamento e não somente como suprimento necessário para o desenvolvimento inicial e sobrevivência da vegetação. Após o período definido como estabelecimento da vegetação (um mês), a partir da irrigação foram monitorados os parâmetros físicos e químicos da qualidade da água escoada pelas unidades experimentais. Buscou-se manter a intensidade da irrigação constante, monitorando-se o tempo de aplicação. Manteve-se a quantidade de irrigação em três vezes semanais e o volume irrigado de $0,5 \mathrm{~L}$ de água destilada durante um minuto. Na próxima fase experimental, as unidades experimentais foram submetidas aos eventos de chuva real para a continuação do monitoramento da qualidade da água escoada. Os dados obtidos desse processo foram analisados tendo como referência os dados provenientes da amostragem dos eventos de precipitação.

\subsubsection{Qualidade da água de escoamento}

A análise qualitativa da água escoada a partir de cada unidade experimental foi realizada por meio da determinação de parâmetros físicos (cor verdadeira, condutividade elétrica, turbidez e sólidos totais) e químicos ( $\mathrm{pH}$, nitrato, fosfato e sulfato). A Figura 4 apresenta o fluxograma da metodologia de pesquisa utilizada para analisar os substratos de telhados verdes nas duas fases experimentais. 
Figura 4: Fluxograma da metodologia de pesquisa utilizada para analisar a água escoada pelos substratos de telhados verdes nas duas fases experimentais.

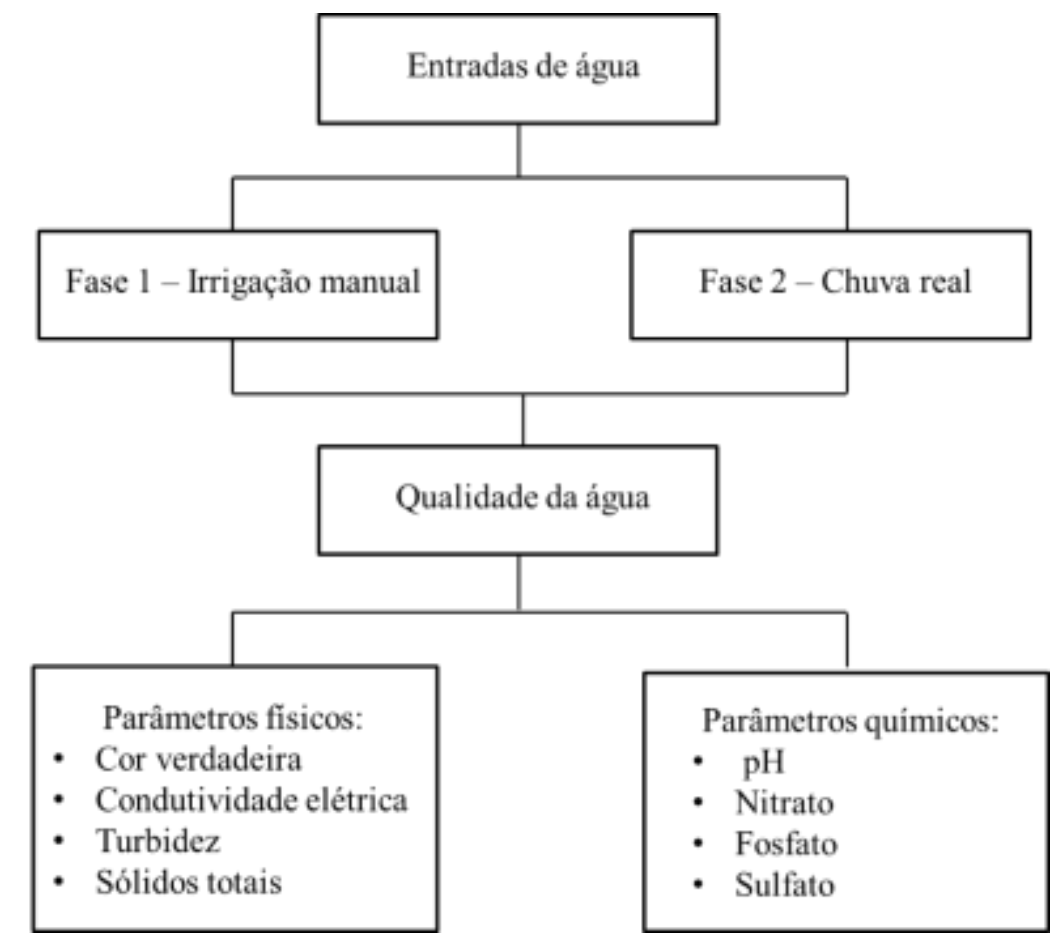

Fonte: Autores.

Para a fase de irrigação, as coletas tiveram periodicidade quinzenal. As amostras de água escoada foram coletadas imediatamente após a irrigação. Posteriormente, na segunda fase do experimento (dados de chuva), as amostras foram coletadas preferencialmente após os eventos chuvosos. Foram realizadas cinco amostragens de água escoada a partir da irrigação manual entre os períodos de julho e setembro de 2018. A partir de janeiro de 2019 foram mensuradas a qualidade da água escoada provenientes de nove eventos de precipitação.

Para os eventos de precipitação foram separadas cinco alíquotas com o mesmo volume (uma para cada repetição) para constituir uma amostra composta. Para garantir a confiabilidade dos resultados obtidos, após cada evento (irrigação manual ou precipitação), os recipientes coletores eram esvaziados e devidamente higienizados.

Para a análise dos íons nitrato, fosfato e sulfato, as amostras foram centrifugadas e posteriormente filtradas em membrana de celulose de $0,45 \mu \mathrm{m}$. Esses ânions foram aferidos por cromatografia iônica, equipamento 883 Basic IC - 863 Compact Auto Sampler. Os métodos de determinação seguiram o Standard Methods for the Examination of Water and Wasterwater (APHA, 2012). O pH foi determinado pelo método potenciométrico com pHmetro (marca Hach), a turbidez por meio do método nefelométrico com turbidímetro (2100 Q Hach), a cor verdadeira foi mensurada por espectrofotometria UVVis (espectrofotômetro DR 6000 - Hach), a condutividade elétrica pelo método eletrométrico com condutivímetro (HQ 40d multiHach) e os sólidos totais foi aferido pelo método gravimétrico.

\subsection{Análise estatística}

Os resultados para os parâmetros físicos e químicos da água escoada para cada tratamento (CON, M e C) foram avaliadas por meio da análise de variância (ANOVA one way) seguida de um teste post hoc de Tukey ( $\alpha=5 \%$ ). Os dados e os resíduos, foram verificados quanto às pressuposições de normalidade pelo teste de Kolmogorov-Smirnov ( $\alpha=5 \%$. Se as 
premissas para os testes paramétricos não fossem atendidas, a transformação logarítmica foi utilizada para homogeneizar as variâncias ou para normalizar a distribuição dos dados.

\section{Resultados e Discussão}

\subsection{Propriedades físicas e químicas dos substratos}

O tamanho das partículas dos substratos foi fixado entre $0,25-2 \mathrm{~mm}$ por meio peneiras granulométricas com malhas desejadas. A variação de massa de cada granulometria não foi controlada. Os componentes do substrato eram de tamanhos similares, o que era intencional, com distribuição de massa diferente, com a intenção de alterar o volume de ar e água mantido pelo substrato final do telhado verde, conforme menciona Vijayaraghavan e Raja (2014). A Figura 5 apresenta a distribuição do tamanho das partículas do substrato no início do estudo.

Figura 5: Distribuição do tamanho das partículas do substrato no início do estudo. Os substratos foram rastreados usando peneiras com aberturas de 0,$25 ; 0,5 ; 1$ e $2 \mathrm{~mm}$.

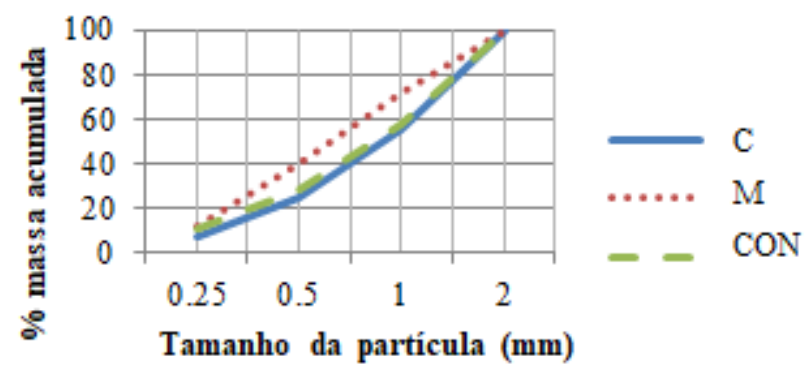

Fonte: Dados da pesquisa.

Conforme observa-se na Figura 5, em termos de distribuição de tamanho de partícula, o substrato M apresentou uma porcentagem maior de partículas menores, com aproximadamente $60 \%$ das partículas variando entre 0,25 e $0,75 \mathrm{~mm}$, enquanto os substratos CON e C apresentaram cerca de $40 \%$ de partículas com a mesma faixa de granulometria.

Para entender o papel dos componentes do substrato de telhado verde na qualidade da água escoada, avaliou-se as características físicas e químicas dos três substratos antes do plantio e os resultados são apresentadas na Tabela 1.

Os valores obtidos para o $\mathrm{pH}$ e densidade foram similares para os três tipos de substratos. O substrato $\mathrm{M}$ apresentou valores mais elevados de retenção de água, turbidez e cor verdadeira. Os elevados valores de turbidez e cor verdadeira podem indicar uma maior liberação de nutrientes, assim como a maior retenção de água pode estar relacionado como a retenção na matéria orgânica proveniente do malte de cevada (Vacari et al., 2019, Vacari et al., 2020). A condutividade elétrica foi maior para o substrato $\mathrm{C}$, que também apresentou maior valor para a CTC. Os substratos $\mathrm{M}$ e $\mathrm{C}$ não apresentaram valores de concentração de sulfato. A utilização de matéria orgânica em substratos de telhados verdes mantém uma boa estrutura do solo, aumenta a capacidade de troca catiônica, melhora a retenção de água e fornece nutrientes para a vegetação (Nagase \& Dunnett, 2011). O volume armazenado de água no substrato promove a disponibilidade de água para as plantas sem ter alto gasto de energia para consumi-la (Vijayaraghavan \& Raja, 2014). 
Tabela 1: Valores médios para os parâmetros físicos e químicos do substrato antes do plantio.

\begin{tabular}{lllll}
\hline Parâmetro & Unidade & CON & M & C \\
\hline Tamanho da partícula & $\mathrm{mm}$ & $0,25-2$ & $0,25-2$ & $0,25-2$ \\
Densidade & $\mathrm{g} / \mathrm{cm}^{3}$ & $0,364 \pm 0,020$ & $0,367 \pm 0,013$ & $0,313 \pm 0,010$ \\
Retenção de água & $\%$ & $33,5 \pm 1,32$ & $43,7 \pm 2,52$ & $32 \pm 1,73$ \\
Condutividade elétrica & $\mu \mathrm{S} / \mathrm{cm}$ & $116.27 \pm 2,17$ & $108,37 \pm 1,96$ & $141,47 \pm 6,66$ \\
$\mathrm{pH}^{*}$ & & $7,37 \pm 0,18$ & $7,22 \pm 0,095$ & $7,14 \pm 0,23$ \\
Turbidez & $103,77 \pm 44,39$ & $156,34 \pm 13,58$ & $93,50 \pm 11,87$ \\
Cor verdadeira* & $\mathrm{NTU}$ & $953,34 \pm 162,89$ & $2386,67 \pm 240,07$ & $946,67 \pm 75,05$ \\
CTC $^{*}$ & $\mathrm{~nm}$ & 225 & 270 & 330 \\
Nitrato $^{*}$ & $\mathrm{Mmol} / \mathrm{Kg}^{-1}$ & $0,9 \pm 0,28$ & $1,35 \pm 0,21$ & $0,47 \pm 0,15$ \\
Fosfato $^{*}$ & $\mathrm{mg} / \mathrm{L}$ & $5,9 \pm 0,40$ & $3,33 \pm 0,51$ & $5,5 \pm 0,52$ \\
Sulfato* $^{*}$ & $\mathrm{mg} / \mathrm{L}$ & $1 \pm 0,25$ & $\mathrm{nd}$ & $\mathrm{nd}$ \\
\hline
\end{tabular}

${ }^{*}$ Resultados obtidos a partir da análise de solubilizados dos substratos. nd = não detectado

Fonte: Dados da pesquisa.

\subsection{Qualidade da água escoada pelos módulos experimentais}

A Tabela 2 apresenta as características dos nove eventos de precipitação monitorados, incluindo os dias sem chuva anterior ao evento, chamados de período seco antecedente. As precipitações variaram de 14,1 a 68,5 mm.

Tabela 2: Especificações dos eventos de precipitação monitorados.

\begin{tabular}{ccccccccc}
\hline Evento & 1 & 2 & 3 & 4 & 5 & 6 & 7 & 8 \\
\hline Data (ano 2019) & $20 / 01$ & $09 / 02$ & $17 / 02$ & $20 / 02$ & $22 / 02$ & $17 / 03$ & $19 / 03$ & $02 / 04$ \\
Precipitação (mm) & 25,5 & 20,2 & 14,1 & 40,5 & 35,3 & 14,5 & 23,5 & 46 \\
PS (dias)* & 1 & 0 & 0 & 0 & 1 & 0 & 1 & 1 \\
\hline
\end{tabular}

*PS: período seco antecedente ao evento de precipitação.

Fonte: Dados da pesquisa.

As Tabela 3 e 4 apresentam as concentrações médias dos parâmetros de qualidade da água escoada para os diferentes tipos de substratos de telhados verdes analisados na fase de irrigação manual e chuva real, respectivamente.

A qualidade da água escoada pelos módulos experimentais de telhados verdes teve grande variabilidade quando comparada às duas fases do experimento. As concentrações dos parâmetros de condutividade elétrica, turbidez e cor verdadeira, foram superiores quando as unidades experimentais estavam recebendo a irrigação manual (Tabelas 3 e 4 ). Provavelmente, os maiores níveis dos parâmetros mencionados podem estar relacionados com o fato de ter sido a fase inicial do experimento, com maior quantidade de materiais e compostos sendo carreados pelo fluxo de água. 
Tabela 3: Comparação da concentração média dos parâmetros de qualidade da água escoada para os diferentes tipos de substratos de telhados verdes analisados na fase de irrigação.

\begin{tabular}{lllll}
\hline Parâmetro $^{*}$ & Unidade & CON & M & C \\
\hline Condutividade elétrica & $\mu \mathrm{S} / \mathrm{cm}$ & $1415,72 \pm 643,43^{\mathrm{a}}$ & $3693,48 \pm 1088,48^{\mathrm{b}}$ & $1185,52457,21^{\mathrm{a}}$ \\
$\mathrm{pH}$ & - & $7,27 \pm 0,30^{\mathrm{a}}$ & $6,77 \pm 0,39^{\mathrm{b}}$ & $7,53 \pm 0,23^{\mathrm{c}}$ \\
Turbidez & $\mathrm{NTU}$ & $52,70 \pm 23,86^{\mathrm{a}}$ & $14,72 \pm 10,62^{\mathrm{b}}$ & $58,61 \pm 25,76^{\mathrm{a}}$ \\
Cor verdadeira & $\mathrm{uH}$ & $1480 \pm 650,89^{\mathrm{a}}$ & $475,2 \pm 251,68^{\mathrm{b}}$ & $1348,8 \pm 493,43^{\mathrm{a}}$ \\
Nitrato & $\mathrm{mg} / \mathrm{L}$ & $12,41 \pm 3,64^{\mathrm{a}}$ & $25,67 \pm 6,67^{\mathrm{b}}$ & $4,91 \pm 9,47^{\mathrm{a}}$ \\
Fosfato & $\mathrm{mg} / \mathrm{L}$ & $5,56 \pm 2,00^{\mathrm{a}}$ & $4,45 \pm 3,92^{\mathrm{b}}$ & $6,88 \pm 1,85^{\mathrm{a}}$ \\
Sulfato & $\mathrm{mg} / \mathrm{L}$ & $7,12 \pm 2,42^{\mathrm{a}}$ & $5,48 \pm 5,49^{\mathrm{a}}$ & $4,54 \pm 3,29^{\mathrm{b}}$ \\
\hline
\end{tabular}

*Os valores seguidos por diferentes letras dentro de uma linha para cada tipo de substrato referem-se a diferenças estatisticamente significantes pelo teste de Tukey, $\alpha=5 \%$.

Fonte: Dados da pesquisa.

Tabela 4: Comparação da concentração média dos parâmetros de qualidade da água escoada para os diferentes tipos de substratos de telhados verdes analisados na fase de chuva real.

\begin{tabular}{lllll}
\hline Parâmetro* $^{*}$ & Unidade & CON & M & C \\
\hline Condutividade elétrica & $\mu \mathrm{S} / \mathrm{cm}$ & $1050,31 \pm 378,66^{\mathrm{a}}$ & $1164,79 \pm 204,89^{\mathrm{a}}$ & $1018,10 \pm 195,39^{\mathrm{a}}$ \\
$\mathrm{pH}$ & - & $7,64 \pm 0,89^{\mathrm{a}}$ & $7,41 \pm 0,94^{\mathrm{a}}$ & $7,46 \pm 0,76^{\mathrm{a}}$ \\
Turbidez & $\mathrm{NTU}$ & $10,27 \pm 4,57^{\mathrm{a}}$ & $12,28 \pm 4,36^{\mathrm{a}}$ & $11,23 \pm 3,81^{\mathrm{a}}$ \\
Cor verdadeira & $\mathrm{uH}$ & $491,79 \pm^{\mathrm{a}}$ & $717,14^{\mathrm{b}}$ & $621,79^{\mathrm{b}}$ \\
Nitrato & $\mathrm{mg} / \mathrm{L}$ & $24,45 \pm 26,43^{\mathrm{a}}$ & $16,05 \pm 11,85^{\mathrm{a}}$ & $8,71 \pm 8,29^{\mathrm{b}}$ \\
Fosfato & $\mathrm{mg} / \mathrm{L}$ & $8,31 \pm 4,54^{\mathrm{a}}$ & $7,15 \pm 2,92^{\mathrm{a}}$ & $7,88 \pm 3,15^{\mathrm{a}}$ \\
Sulfato & $\mathrm{mg} / \mathrm{L}$ & $3,01 \pm 1,67^{\mathrm{a}}$ & $2,07 \pm 0,84^{\mathrm{a}}$ & $1,58 \pm 0,77^{\mathrm{b}}$
\end{tabular}

*Os valores seguidos por diferentes letras dentro de uma linha para cada tipo de substrato referem-se a diferenças estatisticamente significantes pelo teste de Tukey, $\alpha=5 \%$.

Fonte: Dados da pesquisa.

O substrato M apresentou valores maiores para a CE durante a irrigação manual, com média de 3693,48 $\mu \mathrm{S} / \mathrm{cm}$. O substrato $\mathrm{C}$ apresentou a menor média para CE diferindo estatisticamente do substrato $\mathrm{M}(\mathrm{p}=0,0001)$, porém não diferiu do substrato COM (Tabela 3). Para os valores de CE na fase de chuva real, o substrato C apresentou a menor média, entretanto não foram observadas diferenças estatísticas entre os três substratos (Tabela 4). Os elevados níveis de condutividade elétrica provavelmente estão associados com a lixiviação de íons dos substratos de telhados verdes. Tal situação foi verificada por Liberalesso (2018), que observou aumento gradativo da condutividade elétrica conforme a adição de casca de arroz carbonizada na mistura do substrato, assim como aumento nos teores de fósforo total.

Quanto ao parâmetro turbidez, para os resultados provenientes do escoamento da água da chuva, não houve diferença significativa entre os substratos analisados (Tabela 4). Em contrapartida, quando a entrada de água se deu por irrigação manual o substrato M exibiu média significativamente inferior ( $\mathrm{p}=0,0001)$, sendo 14,7 NTU, em comparação com as médias dos outros dois substratos (Tabela 3).

Para a cor verdadeira, o substrato CON obteve a menor média com valor de 491,8 uH (p =0,007) durante os eventos chuvosos. Entretanto, na fase de irrigação manual, o substrato M obteve média de 475,2 uH, valor significativamente inferior 
aos outros meios de crescimento $(\mathrm{p}=0,0001)$, que não diferiram entre si $(\mathrm{p}=0,63)$. O substrato em telhados verdes novos ainda possui grande concentração de nutrientes e matéria orgânica o que pode explicar o fato dos elevados valores de cor verdadeira (Rowe, 2011). Vacari et al. (2020), também constatou altos valores de cor verdadeira, com variação de até 492 uH para módulos de telhados verdes com seis meses de instalação.

Para os valores de $\mathrm{pH}$, durante a fase de irrigação, as médias foram estatisticamente diferentes $(\mathrm{p}=0,0001)$, sendo 6,77 para o substrato M, 7,27 para o COM e 7,53 para o substrato C (Tabela 3). Tal diferença pode estar relacionada com os compostos orgânicos e sua possível degradação, provenientes dos bagaços de malte de cevada e cana-de-açúcar. Já os valores obtidos durante os eventos chuvosos variaram de 7,41 a 7,46, não apresentando diferença estatística entre si (Tabela 4), e indicado a estabilização dos materiais adicionados ao substrato. Cada grupo de telhado verde forneceu valores de pH próximos da neutralidade, o que é benéfico para o ambiente, principalmente no que se refere à neutralização da chuva ácida nos grandes centros urbanos (Hashemi et al., 2015). Uma possibilidade para os valores de pH serem mais próximos da neutralidade é a lixiviação de cátions formadores de bases nos substratos, como $\mathrm{Ca}^{2+}, \mathrm{Mg}^{2+}, \mathrm{K}^{+} \mathrm{e} \mathrm{Na}^{+}$, o que resulta em um aumento no valor do pH da água escoada (Chen et al., 2018).

Nas duas fases do experimento, as concentrações de $\mathrm{NO}_{3}^{-}$foram mais baixas para o substrato C, com diferença estatística dos demais substratos para a fase de chuva real $(\mathrm{p}=0,02)$. Para a irrigação manual o substrato $\mathrm{C}$ diferiu significativamente apenas do substrato $\mathrm{M}(\mathrm{p}=0,0001)$ (Tabelas 3 e 4). O desempenho do substrato C na redução da lixiviação deste íon, durante os eventos de precipitação, indicam potencialidade para a utilização desse componente em substratos de telhado verde, com a finalidade de diminuir as concentrações de $\mathrm{NO}_{3}^{-}$na água escoada.

Beck et al. (2011) analisaram a adição de biocarvão em substratos de telhados verdes e verificaram que os níveis de nitrato foram significativamente inferiores quando comparados ao escoamento das bandejas experimentais contendo apenas o solo controle. Teemusk \& Mander (2007) complementam que a concentração de nitrogênio, nas suas diversas formas, tende a

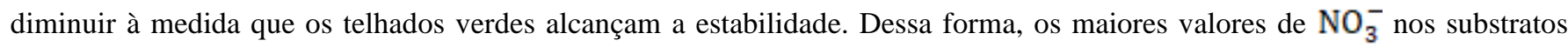
COM e C na fase 2, eventos chuvosos, podem estar relacionados com a ciclagem de nitrogênio, possivelmente pela intensificação do processo de nitrificação (Chen et al., 2018).

A concentração de $\mathrm{PO}_{4}^{3-}$ foi menor para o substrato com a adição do bagaço de malte na fase $1(4,45 \mathrm{mg} / \mathrm{L})(\mathrm{Tabela}$ 3). Percebe-se que houve um acréscimo na lixiviação de íons fosfato durante a fase de chuva real. A intensidade e duração do evento chuvoso influência o carreamento de compostos a partir do substrato (Rowe, 2011) e isso pode ter contribuído para o aumento das concentrações de $\mathrm{PO}_{4}^{3-}$ para as amostras de água escoada na fase 2. Pessoa (2016) também encontrou concentrações semelhantes de fosfato em um telhado verde submetido a eventos de precipitação, variando de 4,69 a 7,13 mg/L. O autor verificou que houve um substancial incremento de fosfato nas águas pluviais após sua passagem pela cobertura verde.

Com relação à concentração de sulfato, na fase de irrigação manual os valores foram mais elevados do que para a etapa de chuva real. O substrato $\mathrm{C}$ apresentou concentrações inferiores de sulfato comparadas aos outros tratamentos, tanto na irrigação manual, com média de 4,54 mg/L, quanto na fase de chuva real, com média de $1,58 \mathrm{mg} / \mathrm{L}$. Os valores de $\mathrm{SO}_{4}^{2-}$ para os substratos de telhados verdes propostos foram inferiores aos valores encontrados por Pessoa (2016), que verificou concentrações que variaram de 1,26 a 22,68 mg/L num período de sete meses. Teemusk e Mander (2011) observaram que os telhados verdes possuíam maiores concentrações de sulfatos devido a presença de agregado leve e de baixa de densidade, como por exemplo, a vermiculita expandida. 
Os resultados indicaram que só houve redução significativa com relação aos ânions $\mathrm{NO}_{3}^{-} \mathrm{e} \mathrm{SO}_{4}^{2-}$, sendo o substrato C com melhor desempenho na retenção de nutrientes. O substrato controle apresentou melhores resultados quanto à turbidez e a cor verdadeira. Os outros parâmetros analisados não tiveram diferença significativa entre os tratamentos.

Segundo Rowe (2011) diversos poluentes são encontrados em maiores concentrações em telhados verdes jovens, pois o solo ainda está concentrado e a vegetação ainda não completamente está estabelecida. Portanto, cabe ressaltar a importância do monitoramento por um período de tempo superior, a fim de se verificar as modificações decorrentes do processo de decomposição da matéria orgânica dos substratos e sua influência no tempo.

\section{Conclusão}

Os efeitos de substratos acrescidos de bagaço de malte e bagaço de cana-de-açúcar na qualidade da água escoada por módulos experimentais de telhados verdes foram monitorados e comparados no presente estudo. Os resultados indicaram que houve redução significativa dos ânions $\mathrm{NO}_{3}^{-}$e $\mathrm{SO}_{4}^{2-}$, na água escoada pelo substrato contendo o bagaço de cana-de-açúcar, se destacando, portanto, na retenção de nutrientes. O substrato controle apresentou melhores resultados quanto à turbidez e a cor verdadeira. A distribuição e o impacto da água no meio de crescimento das unidades experimentais interferem na lixiviação de nutrientes dos substratos, como observou-se entre a fase 1 e 2 desse experimento.

Os materiais adsorventes de baixo custo, como o bagaço de cana-de-açúcar, que apresentou resultados satisfatórios na diminuição da lixiviação de alguns íons são potenciais componentes para substratos de telhados verdes. Assim, recomenda-se o aprimoramento de estudos sobre a utilização de bagaço de cana-de-açúcar em substratos para as coberturas verdes.

\section{Agradecimentos}

O presente trabalho foi realizado com apoio da Coordenação de Aperfeiçoamento de Pessoal de Nível Superior Brasil (CAPES) - Código de Financiamento 001

\section{Referências}

Alsup, S. E., Ebbs, S. D., Battaglia, L. L., \& Retzlaff, W. A. (2011). Heavy metals in leachate from simulated green roof systems. Ecoloogical Engineering, $37(11), 1709-1717$.

Apha - American Public Health Association; American Water Works Association; Water Environment Federation. (2012). Standard methods for the examination of water and wasterwater. $22^{\mathrm{a}} \mathrm{Ed}$. Washington: APHA/ AWWA/WEF.

Beck, D. A., Jhonson, G. R., \& Spolek, G. A. (2011). Amending greenroof soil with biochar to affect runoff water quantity and quality. Environmental Pollution, 159(8-9), 2111-2118.

Berndtsson, J. C., Emilsson, T. T., \& Bengtsson, L. (2006). The influence of extensive vegetated roofs on runoff water quality. Science of the Total Environment, 355(1-3), 48-63.

Berndtsson, J. (2010). Green roof performance towards management of runoff water quantity and quality: A review. Ecological Engineering, 36(4), 351-360.

Brasil. Ministério da Agricultura, Pecuária e Abastecimento - MAPA. (2017). Manual de métodos analíticos oficiais para fertilizantes e corretivos. Brasília: Secretaria de Defesa Agropecuária.

Buffam, I., \& Mitchell, M. E. (2015). Nutrient cycling in green roof ecosystems. In: Sutton, R. Green roof ecosystems. Switzerland: Springer International Publishing, 107-137.

Chen, C. F., Kang, S. F., \& Lin, J. H. (2018). Effects of recycled glass and different substrate materials on the leachate quality and plant growth of green roofs. Ecological Engineering, 112, 10-20.

Cuiabá. Secretaria Municipal de Desenvolvimento Urbano - SMDU. (2012). Perfil socioeconômico de Cuiabá, 5. Central de Texto.

Durhman, A. K., Rowe, D. B., Rugh, C. L. (2007). Effect of substrate depth on initial growth, coverage, and survival of 25 succulent green roof plant taxa. HortScience, 42(3), 588-595.

Embrapa - Empresa Brasileira de Pesquisa Agropecuária. (2017). Manual de métodos de análise de solo. (3a ed.). Brasília: EMBRAPA. 
Ferreira, P. P. L., Braga, R. M., Teodoro, N. M. A., Melo, V. R. M., Melo, D. M. A., \& Melo, M. A. F. (2015). Adsorção de $\mathrm{Cu}^{2+} \mathrm{e} \mathrm{Cr}^{3+} \mathrm{em}^{2}$ efluentes líquidos utilizando a cinza do bagaço da cana-de-açúcar. Cerâmica, 61(360), 435-441.

Hashemi, S. S. G., Mahmud, H. B., \& Ashraf, M. A. (2015). Performance of green roofs with respect to water quality and reduction of energy consumption in tropics: a review. Renewable and Sustainable Energy Reviews, 52, 669-679.

Johnston, J; \& Newton, J. (2004). Building Green: a guide to using plants on roofs, walls and pavements. Greater Authority.

Liberalesso, T. (2018). Telhados verdes extensivos: influência da composição do substrato na retenção hídrica e no desenvolvimento da vegetação. Dissertação (Mestrado em Engenharia Ambiental). Programa de Pós-Graduação em Engenharia Ambiental. Santa Maria.

Mato Grosso, Secretaria de Estado do Meio Ambiente - SEMA. (2016). Superintendência de Regularização e Monitoramento Ambiental. Relatório de Monitoramento da Qualidade da Água da Região Hidrográfica do Paraguai - 2012-2014. Cuiabá: SEMA/MT.

Nagase, A., \& Dunnet, N. (2012). Amount of water runoff from different vegetation types on extensive green roofs: Effects of plant species, diversity and plant structure. Landscape and Urban Planning, 104(3-4), 356-363.

Niemelã, J. (2014). Ecology of urban green spaces: the way forward in answering major research questions. Landscape Urban Planning, 125, 298-303.

Noya, M. G., Cuquel, F. L., Schafer, G., \& Armindo, R. A. (2017). Substrates for cultivating herbaceous perennial plants in extensive green roofs. Ecological Engineering, 102, 662-669.

Pereira, A. S., Shitsuka, D. M., Parreira, F. J., \& Shitsuka, R. (2018). Metodologia da pesquisa Científica. UFSM, https://repositorio.ufsm.br/bitstream/há ndle/1 /15824/Lic_Computacao_Metodologia-Pesquisa-Cientifica.pdf?sequence=1

Pessoa, J. O. (2016). Quantidade e qualidade de águas pluviais escoadas a partir de telhados verdes extensivos em Santa Maria - RS. Dissertação (Mestrado em Engenharia Ambiental). Programa de Pós-Graduação em Engenharia Ambiental. UFMS: Santa Maria.

Reis, H. C. O., Cossolin, A. S., Santos, B. A. P., Castro, K. C., Pereira, G. M., Silva, V. C., Sousa Jr, P. T., Dall’Oglio, E. L., Vasconcelos, L. G., \& Morais, E. B. (2018). Malt bagasse waste as biosorbent for Malachite green: an ecofriendly approach for dye removal from aqueous solution. World Academy of Science, Engineering and Technology (Online), 12(4), 118-126.

Rincón, C. A., Cuesta, M. P. A., Perez, B. R. (1992). Maníforrajero perenne (Arachis pintoi Krapovickas e Gregory): Una alternativa para ganaderos e agricultores. Bogotá: Instituto Colombiano Agropecuário.

Rowe, D. B. (2011). Green roofs as a means of pollution abatement. Environmental Pollution, 159(8-9), 2100-2110.

Silva, W. L. L., \& Oliveira, S. P. (2012). Modificação das características de adsorção do bagaço de cana para remoção de azul de metileno de soluções aquosas. Scientia Plena, 8(9), 1-9.

Speak, A. F., Rothwell, J. J., Lindley, S. J., \& Smith, C. L. (2014). Metal and nutrient dynamics on an aged intensive green roof. Environmental Pollution, $184,33-43$.

Teemusk, A., \& Mander, U. (2007). Rainwater runoff quantity and quality performance from a greenroof: The effects of short-term events. Ecological Engineering, 30, 271-277.

Vacari, T. C., Lara, J. R., Lima, Z. M., \& Morais, E. B. (2019). Water quantity investigation of simulated green roofs in a tropical climate: influence of vegetation composition. E\&S Engineering and Science, 8(2), 2-14.

Vacari, T. C., Lima, Z. M., \& Morais, E. B. (2020). Qualidade da água escoada por módulos de telhados verdes com diferentes composições de vegetação. In: Silva, C. S. (Org.). Engenharia Sanitária e Ambiental: Tecnologias para a Sustentabilidade 5. Ponta Grossa: Atena Editora, 264-276.

Valls, J. F. M. (19922). Origem do germoplasma de Arachis pintoi disponível no Brasil. In: Reunión Sabanas - De la Red Internacional de Evaluación de Pastos Tropicales. CIAT, p. 81-96.

Vijayaraghavan, K., Joshi, U. M., \& Balasubramanian, R. (2012). A field study to evaluate runoff quality from green roofs. Water Research, 46(4), 13371345 .

Vijayaraghavan, K., \& Raja, F. D. (2014). Design and development of green roof substrate to improve runoff water quality: Plant growth experiments and adsorption. Water Research, 63, 94-101.

Vijayaraghavan, K., \& Joshi, U. M. (2014). Can green roof act as a sink for contaminants? A methodological study to evaluate runoff quality from green roofs. Environmental Pollution, 194, 121-129.

Young, T., Cameron, D. D., Sorrill, J., Edwards, T., \& Phoenix, G. K. (2014). Importance of different components of green roof substrate on plant growth and physiological performance. Urban Forestry \& Urban Greening, 13(3), 507-516.

Wahba, S., Kamil, B., Nassar, K., \& Abdelsalam, A. (2019). Green envelop impact on reducing air temperature and enhancing outdoor thermal comfort in arid climates. Civil Engineering Journal, 5(5), 1124-1135.

Wahba, S. M., Kamel, B. A., Nassar, K. M., \& Abdelsalam, A. S. (2018). Effectiveness of green roofs and green walls on energy consumption and indoor comfort in arid climates. Civil Engineering Journal, 4(10), 2284-2295. 